\title{
Performance evaluation of magnetically driven cytology brush applicable to capsule endoscopy
}

\author{
Kohei Hajima, Maki Yamashita and Takashi Honda* \\ Department of Applied Science for Integrated System Engineering, Kyushu Institute of Technology, \\ Sensuichou, Kitakyushu, Japan
}

\begin{abstract}
This article provides a novel magnetic actuator suitable for brush cytology using capsule endoscopy. Its space-saving mechanism consisting of a brush-tipped elastic wire coupled with a rotatable permanent magnet can be easily assembled in a swallowable capsule. When external magnetic fields are applied, the brush projects outwards with rotation of the magnet, and then collects cells from gastrointestinal tract. The distinctive feature of the proposed actuator is that it can perform four different actuations including projection/pullback/scrape modes for the brush and a rotation mode for the capsule body. Each actuation mode can be selected by the direction of the external magnetic fields. This study has verified that each mode can exhibit the desired performance and determined its driving conditions. Furthermore, an additional small magnet attached to the brush tip has been found to enhance the performance of the scrape mode. The results obtained in this study suggested that the proposed actuator should be a hopeful candidate for cytological diagnosis with capsule endoscopy.
\end{abstract}

Keywords: Brush cytology, capsule endoscope, external magnetic field, magnetic torque, permanent magnet

\section{Introduction}

Over the last decade, capsule endoscopy (CE) has become widespread as an effective procedure inspecting a small intestine. Its function of inspection using a built-in video camera and wireless communication allows a physician to examine in a minimally invasive manner. Only through a video image, however, the physician cannot determine whether the tumor is malignant or benign. To make a correct diagnosis and provide proper treatment, the physician need perform a biopsy or a cytology diagnosis. Thus a great deal of effort is currently being made on next-generation CE with diagnosis functions [1]2]. But there have been very few clinically successful studies so far because conventional techniques can hardly build both an actuator and a power source in the limited inner space.

Recently, preceding attempts have demonstrated that wireless operation using external magnetic fields would be a practical solution for both miniaturization of the actuator and a battery-free structure. For example, additional functions such as navigation, active locomotion and drug delivery for the CE have been proposed [3-5]. They are based on a common concept in which one or a few permanent magnets

${ }^{*}$ Corresponding author: Takashi Honda, Department of Applied Science for Integrated System Engineering, Kyushu Institute of Technology, 1-1 Sensuichou, Kitakyushu 804-8550, Japan. Tel.: +81 93884 3242; Fax: +81 93 884 3242; E-mail: honda@ele.kyutech.ac.jp.

1383-5416/16/\$35.00 (C) 2016 - IOS Press and the authors. All rights reserved

This article is published online with Open Access and distributed under the terms of the Creative Commons Attribution NonCommercial License. 
in the mechanism are directly operated by external magnetic fields. It is reasonable to suppose that this concept will be helpful to add a function of diagnosis.

Based upon this supposition, our early study attempted to assemble a first prototype actuator capable of putting in/out a brush for cytology with the CE [6]. It was designed so that the brush could project out of the capsule when required and collect cells from gastrointestinal (GI) tract. All actuations could be remotely controlled by the external magnetic fields. However, the malfunction of the actuator was often caused by the least shock. In addition, the ability to collect cells was poor only by the simple motion of putting in/out the brush. In order to improve the reliability and performance of brush cytology, we have designed a new mechanism with a magnetic latch and introduced a scrape mode in the present study. This article describes the basic concept of each actuation mode, the device structure and the performance testing results.

\section{Actuation concept}

As a beginning, the basic concept of four actuation modes performed in this study will be described. All modes utilize magnetic torque $\boldsymbol{T}$ acting on a magnet, which is given by the vector product of the magnetic moment $\boldsymbol{M}$ and the external magnetic field $\boldsymbol{H}$.

Figure 1 shows a conceptual figure of a series of actuation modes. A magnetic actuator installed in the capsule consists of a brush-tipped elastic wire attached to a rotatable magnet with a pivot laterally fixed. An arrow of the magnet indicates the magnetic moment direction. Figure 1(a) shows the initial state, in which the wire is slightly wound up and the brush is housed inside the bottom of the capsule. This state is kept unless a magnetic field is applied. A three dimensional coordinate system with $\mathrm{x}, \mathrm{y}$ and $\mathrm{z}$-axes oriented by arrows is assumed as indicated in this figure. The $\mathrm{x}$-axis corresponds to a longitudinal direction along the GI tract.

The sequence of the operation procedure is as follows. Firstly, the capsule must rotate about the x-axis to align the brush position to the tumor. When either a DC or rotating magnetic field is applied in the yz-plane, the capsule rotates due to magnetic torque acting on the magnet as shown in Fig. 1(b). After the alignment is adjusted, a DC magnetic field is applied in the positive $\mathrm{x}$-direction. Then the magnet rotates about the pivot due to magnetic torque, which causes the brush to project outwards as shown in Fig. 1(c). Subsequently, as the magnetic field is switched to a z-axis AC field, the magnet oscillates angularly and then provides a reciprocating linear motion to the brush as shown in Fig. 1(d). Thus, the brush firmly scrapes a surface of GI tract to collect the cells. Finally, the brush is pulled back to the original position by a DC magnetic field in the negative $x$-direction as shown in Fig. 1(e).

\section{Structure}

Figure 2 shows a configuration of a capsule equipped with a magnetically driven cytology brush. The capsule made from clear polystyrene is $27 \mathrm{~mm}$ in length and $10.7 \mathrm{~mm}$ in outer diameter, which is approximately the size of a commercial capsule endoscope. A round-shaped $\mathrm{Nd}-\mathrm{Fe}-\mathrm{B}$ magnet with a diameter of $5 \mathrm{~mm}$ and a height of $3 \mathrm{~mm}$ is mounted on the rear part by a pivot that is laterally fixed at a position offset upward from the center axis of the capsule. An arrow in the magnet indicates the magnetic moment. A super elastic Ni-Ti wire with a diameter of $0.15 \mathrm{~mm}$ is attached to the magnet by a lever arm. A nylon twisted wire brush with a diameter of $2.7 \mathrm{~mm}$ and a length of $10 \mathrm{~mm}$ is fixed to the tip of the Ni-Ti wire and is housed in a guide pipe at the initial state. The initial position of the magnet is 


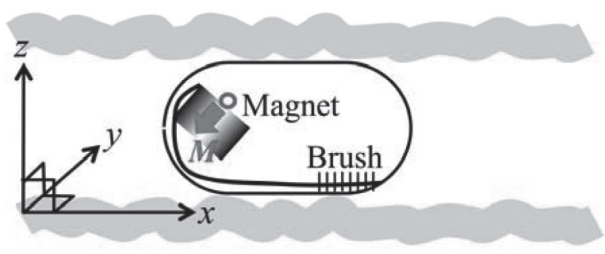

(a) Initial state

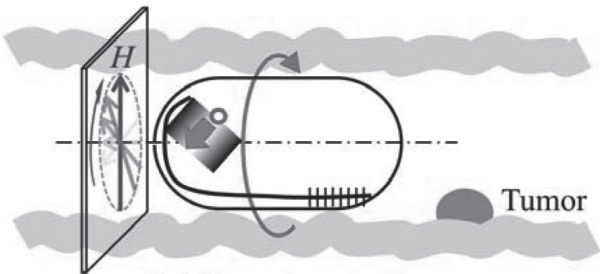

(b) Capsule rotation

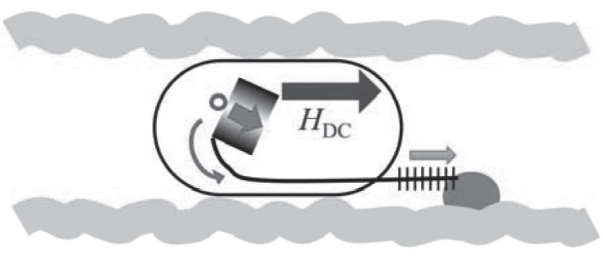

(c) Projection

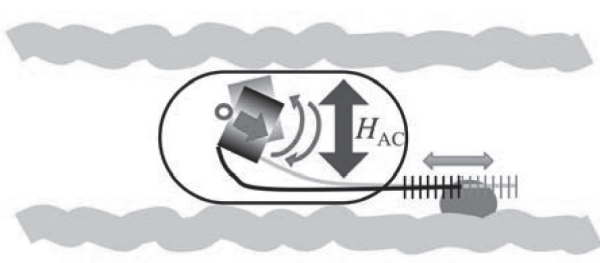

(d) Scrape

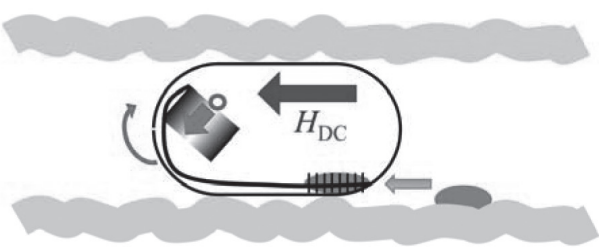

(e) Pullback

Fig. 1. Conceptual figure of a series of actuation modes.

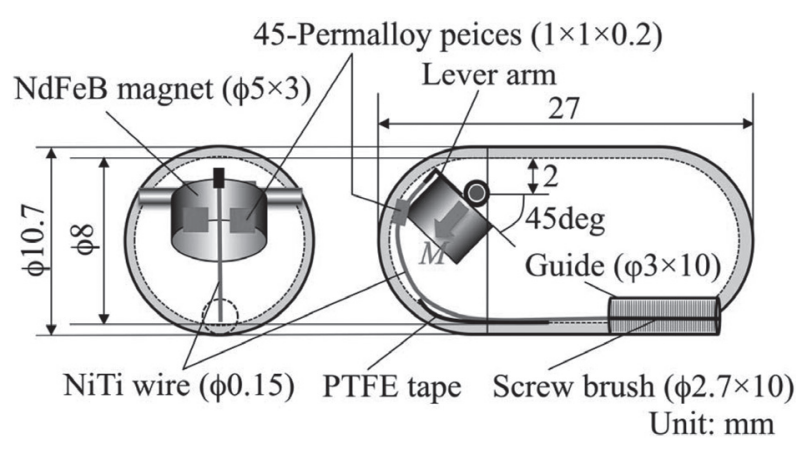

Fig. 2. Configuration of a capsule with a cytology brush.

inclined at 45 degree backward so that the maximum projecting length of the brush is set as $10 \mathrm{~mm}$. This posture is latched by magnetic attractive force between the magnet and two permalloy pieces placed on the inner rear wall of the capsule. In connection with this, a polytetrafluoroethylene (PTFE) tape is stuck on the appropriate part of the inner wall so that the friction between the $\mathrm{Ni}$-Ti wire and the inner wall can be reduced. The specific dimensions of each part are as indicated in Fig. 2. The total weight including the capsule body is approximately $1.7 \mathrm{~g}$.

Figure 3 shows photographs of a cutaway model of the capsule. The upper one is the initial state, and the lower one is the state after projection of the brush. It will be seen that there is a large free space inside the capsule owing to the space saving mechanism. 


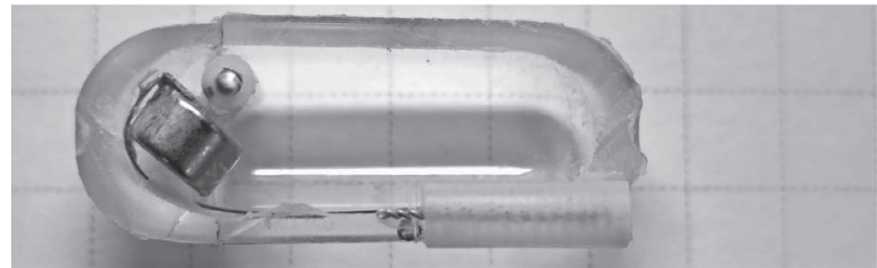

(a) Initial state

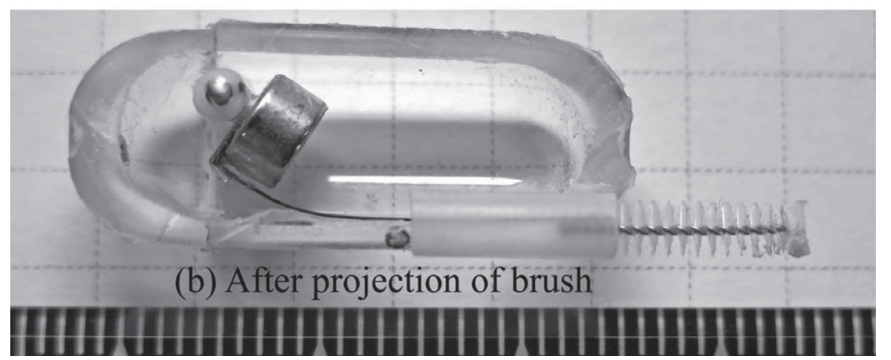

Fig. 3. Photograph of a cutaway model of a capsule equipped with a magnetically driven cytology brush.

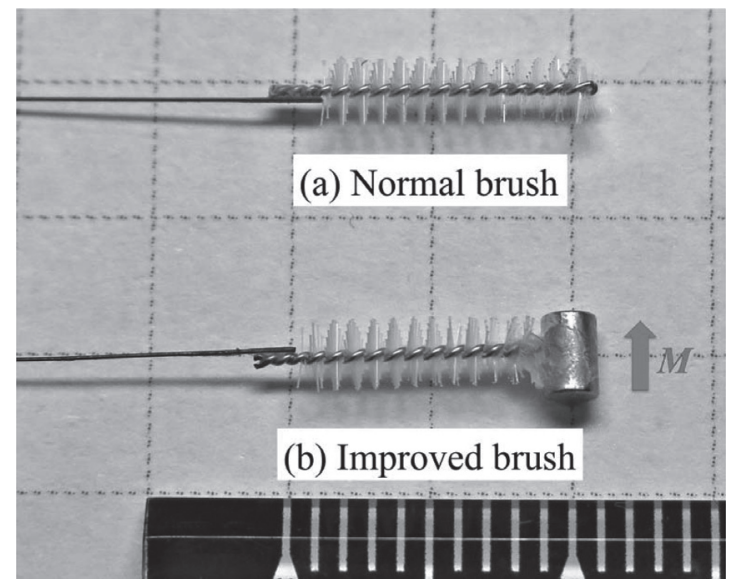

Fig. 4. Two kinds of nylon twisted wire brushes.

Furthermore, we prepared an improved brush in addition to the normal one stated above. It has an additional small Nd-Fe-B magnet with a diameter of $2 \mathrm{~mm}$ and a height of $3 \mathrm{~mm}$ on the tip as shown in Fig. 4(b). The magnetic moment of the small magnet is the opposite direction of the large one. When the DC magnetic field is applied in the positive $\mathrm{x}$-direction in the projection state, the torque acting on the small magnet pushes down the tip of the brush. Therefore, if the same thing is done during the operation on the scrape mode, the brush will scrape the GI tract with pressing force as shown in Fig. 5 and collect the cells more efficiently.

\section{Experiments}

Figure 6 shows an experimental setup for magnetic operation. All operations were performed using cubic 3D Helmholtz coils (Hayama Inc., HHC-15CB-100-3D). The working space where the coils can 


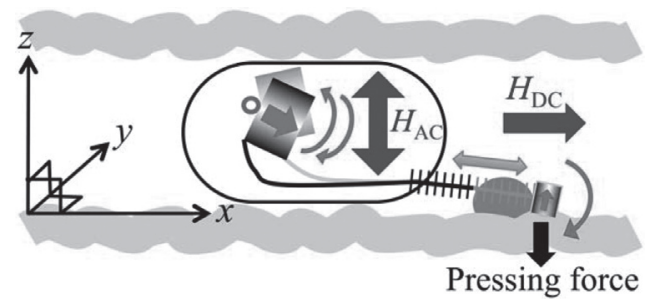

Fig. 5. Scrape mode with pressing force when an improved brush is used.

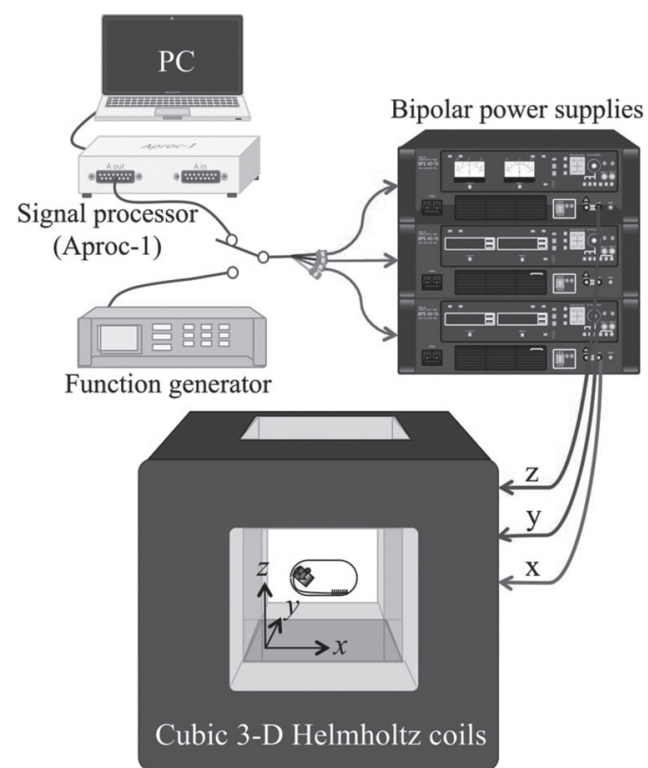

Fig. 6. Experimental setup for magnetic operation.

excite a uniform field is a cube $10 \mathrm{~cm}$ on each side. Each coil is connected to a bipolar power supply (Takasago Ltd., BPS40-15 or BWS40-15), controlled by a function generator or a signal processer (Arima Denshikizai, Aproc-1) edited by a PC. This 3D coil system can generate DC and AC magnetic fields with arbitrary directions. Of course, it can generate rotating magnetic field (RMF) in arbitrary spatial plane. The maximum magnetic field strength generated by this system is approximately $12 \mathrm{kA} / \mathrm{m}$. The capsule was held in a horizontal posture at the center of the 3D coils. Figure 7 shows two types of evaluation environment. When the various forces of the brush were measured, the capsule was fixed on a flat stage top as shown in Fig. 7(a). The forces of the brush were measured using a digital force gauge with a movable stage. When the capsule rotation and the scraping performance were evaluated, the capsule was inserted into a cow's small intestine. At this time, the capsular end with the brush was protruded slightly from the side end of the small intestine as shown in Fig. 7(b). The dynamic motion of the actuator was observed with a digital camcorder and a high-speed video camera.

\section{Results and discussion}

This study has evaluated the performance of the individual modes taking account of an actual operation environment and determined their driving conditions. In our preliminary experiment, the maximum 

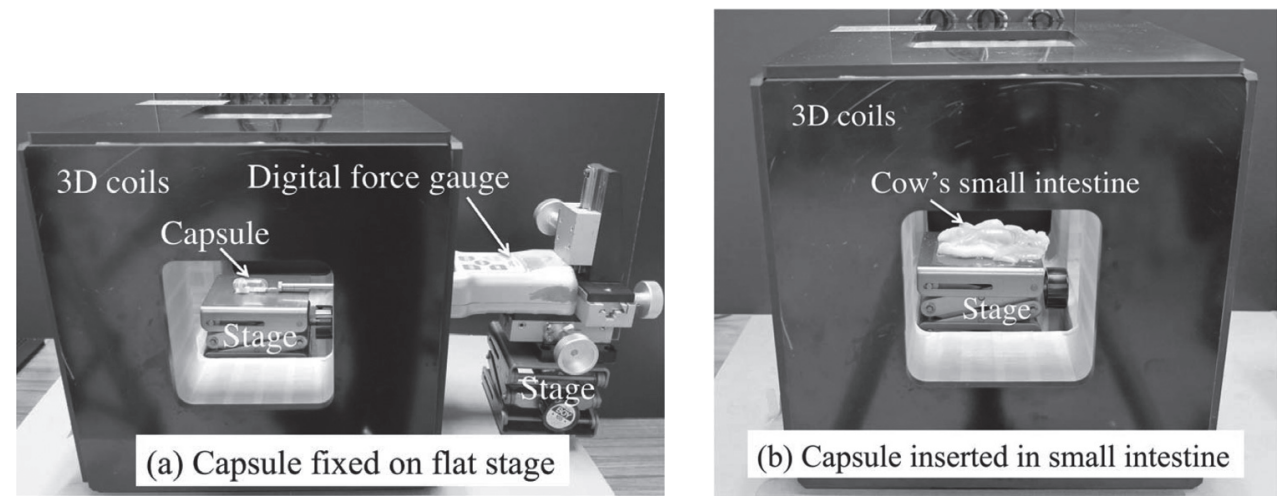

(b) Capsule inserted in small intestine

Fig. 7. Two types of evaluation environment.

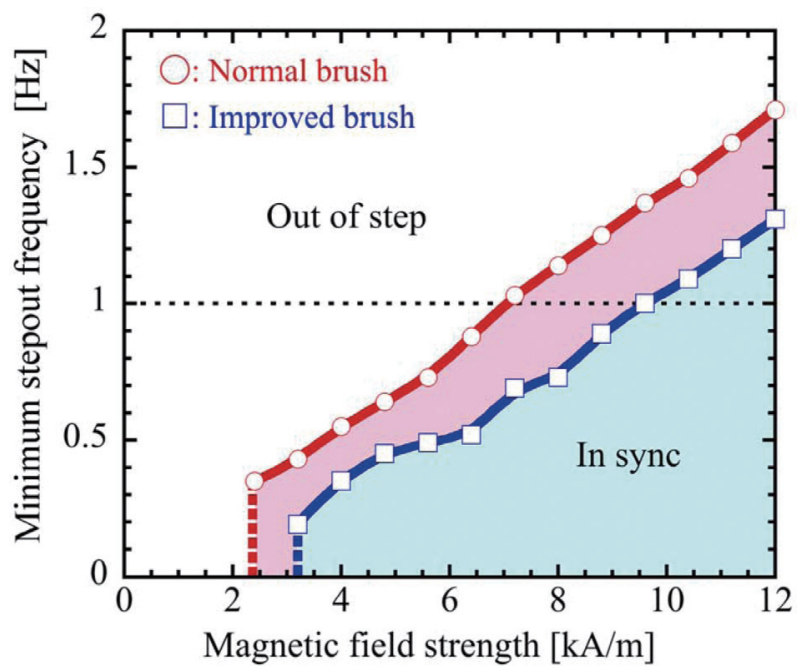

Fig. 8. Minimum step-out frequency of the capsule as a function of the magnetic field strength of RMF in the yz-plane.

static friction between the brush and an inner wall of a fresh cow's small intestine was $13-38 \mathrm{mN}$. In consideration of this friction variation, the target force for the brush actuation was set as more than $40 \mathrm{mN}$ for the projection, pullback and scrape modes.

\subsection{Capsule rotation mode}

The capsule rotation about the $\mathrm{x}$-axis was evaluated by applying a rotating magnetic field (RMF) in the yz-plane as shown in Fig. 1(b), when the capsule was inserted into the cow's small intestine. Fig. 8 shows the minimum frequency causing step-out to the capsule rotation with or without the additional magnet as a function of the magnetic field strength of the RMF. Although the thresholds were observed in low magnetic fields due to the maximum static friction torque, the minimum step-out frequency was nearly proportional to the magnetic field strength. The additional magnet reduced the minimum step-out frequency by $20-40 \%$ because its magnetic moment is opposite direction to the main magnet. Despite such negative affect of the additional magnet, however, both capsules could synchronously rotate without step-out at $1 \mathrm{~Hz}$ that is enough rotational speed to manually adjust the alignment of the brush position. 


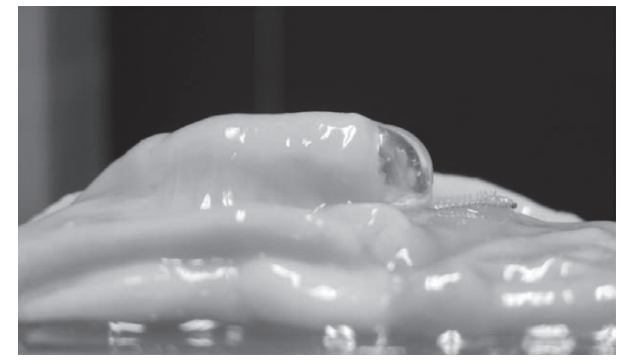

(a) Side view of the capsule inserted in small intestine

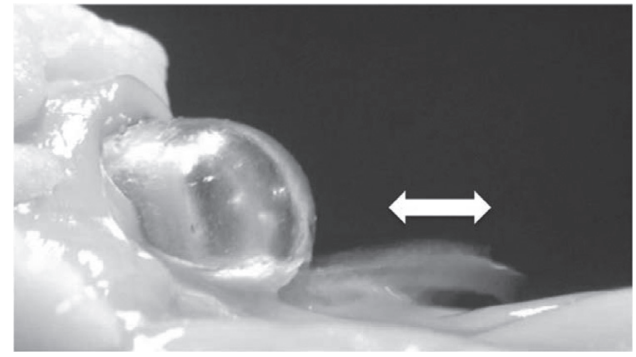

(b) Scraping behavior of the brush

Fig. 9. Side view of the capsule inserted in the small intestine and the actuation behavior of the brush while the $\mathrm{z}$-axis AC magnetic field was applied.

Therefore, the magnetic field strength applied for the capsule rotation mode was determined as $7 \mathrm{kA} / \mathrm{m}$ or more for the normal brush and $9.5 \mathrm{kA} / \mathrm{m}$ or more for the improved one.

\subsection{Projection mode}

In our previous report, the latch at the initial state was based on the friction between the elastic material and the inner wall of the capsule [6]. But malfunction often occurred by shock and the brush projected outwards when not required. As this countermeasure, we have adopted the magnetic latch in this study.

To begin with, the attractive force of the magnet and the permalloy pieces was adjusted to satisfy the following two conditions; the latch passes a shock drop test from a height of $1 \mathrm{~m}$ : the latch can be released by the magnetic field below $12 \mathrm{kA} / \mathrm{m}$. As a result, the attractive force was adjusted to be $104 \mathrm{mN}$, while the DC magnetic field strength in the $\mathrm{x}$-direction required for the latch release was more than $8.7 \mathrm{kA} / \mathrm{m}$.

Once the latch was released, the Ni-Ti wire sprang back into the straight shape, which caused the projection of the whole brush even if the magnetic field was not applied. In addition, the pushing force of the brush by the elastic force was measured to be $46 \mathrm{mN}$ at the projected length of $9 \mathrm{~mm}$ without help by the magnetic field. These results mean that the magnetic field for the projection mode has only to be applied for a short period of time while the latch is released.

\subsection{Scrape mode}

First, the magnetic field strength required for the scrape mode was measured by applying the DC magnetic fields in both positive and negative z-direction. The pushing/pulling forces exceeded the target value of $40 \mathrm{mN}$, when the DC magnetic field was $5 \mathrm{kA} / \mathrm{m}$ or more in both directions. Next, the z-axis AC magnetic field was applied as shown in Fig. 1(d). A reciprocating linear motion of the brush was observed as expected. The reciprocating stroke was $2.5-3.2 \mathrm{~mm}$ in the air while the magnetic field of $6 \mathrm{kA} / \mathrm{m}$ was applied at DC-30 Hz.

Based on the above results, the scraping performance using the normal brush was examined in the cow's small intestine. Figure 9 shows a side view of the capsule inserted in the small intestine (Fig. 9(a)) and the actuation behavior of the brush (Fig. 9(b)) while the z-axis AC magnetic field of $6 \mathrm{kA} / \mathrm{m}$ at $15 \mathrm{~Hz}$ was applied. A piece of opened up small intestine was spread under the brush so that the brush could scrape on parallel to the surface. The reciprocating motion of the brush was observed on the surface of the small intestine as well. However, the scraped amount of the specimen was too small since the brush was bounced on the surface of the small intestine. 


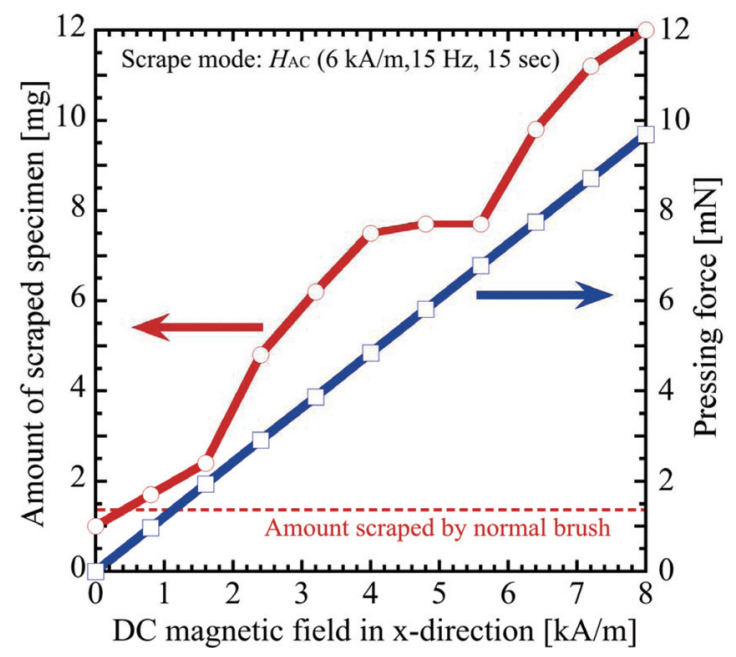

Fig. 10. Mass of specimen scraped by the improved brush and pressing force of the brush as a function of the DC magnetic field in the positive x-direction. Amount scraped by the normal brush shown by a broken line is $1.4 \mathrm{mg}$.
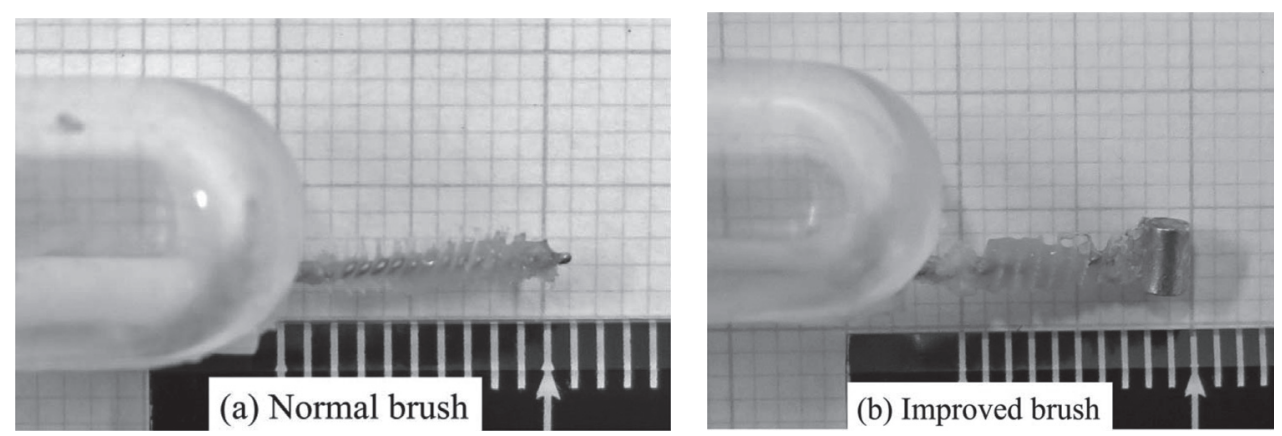

Fig. 11. Appearance of two kinds of brushes after scraping.

In order to improve the scrape performance, the brush was exchanged for the improved type and then the DC magnetic field was applied in the positive x-direction during the scrape mode. The mass of the specimens scraped by the brush was measured after the z-axis AC magnetic field of $6 \mathrm{kA} / \mathrm{m}$ at $15 \mathrm{~Hz}$ was applied for 15 seconds. Figure 10 shows the mass of the specimen as a function of the DC magnetic field in the positive $\mathrm{x}$-direction. In this figure, the pressing force of the tip of the improved brush is plotted as well. The amount of the specimen scraped by the normal brush was only $1.4 \mathrm{mg}$. On the other hand, the amount scraped by the improved one was remarkably improved by increasing the pressing force by increasing the $\mathrm{x}$-axis DC magnetic field. Figure 11 shows the comparison of the appearance of two kinds of brushes after scraping. Here, the $\mathrm{x}$-axis DC magnetic field was $4 \mathrm{kA} / \mathrm{m}$ for the improved one. It is visually confirmed that there is large difference in both collected specimens. As a result of these, the brush with the additional magnet is found to be significantly effective for the scrape mode.

\subsection{Pullback mode}

The pullback test was carried out while the load of $40 \mathrm{mN}$ in the positive $\mathrm{x}$-direction was imposed on the brush and the DC magnetic field was applied in the negative x-direction. When the magnet winds 

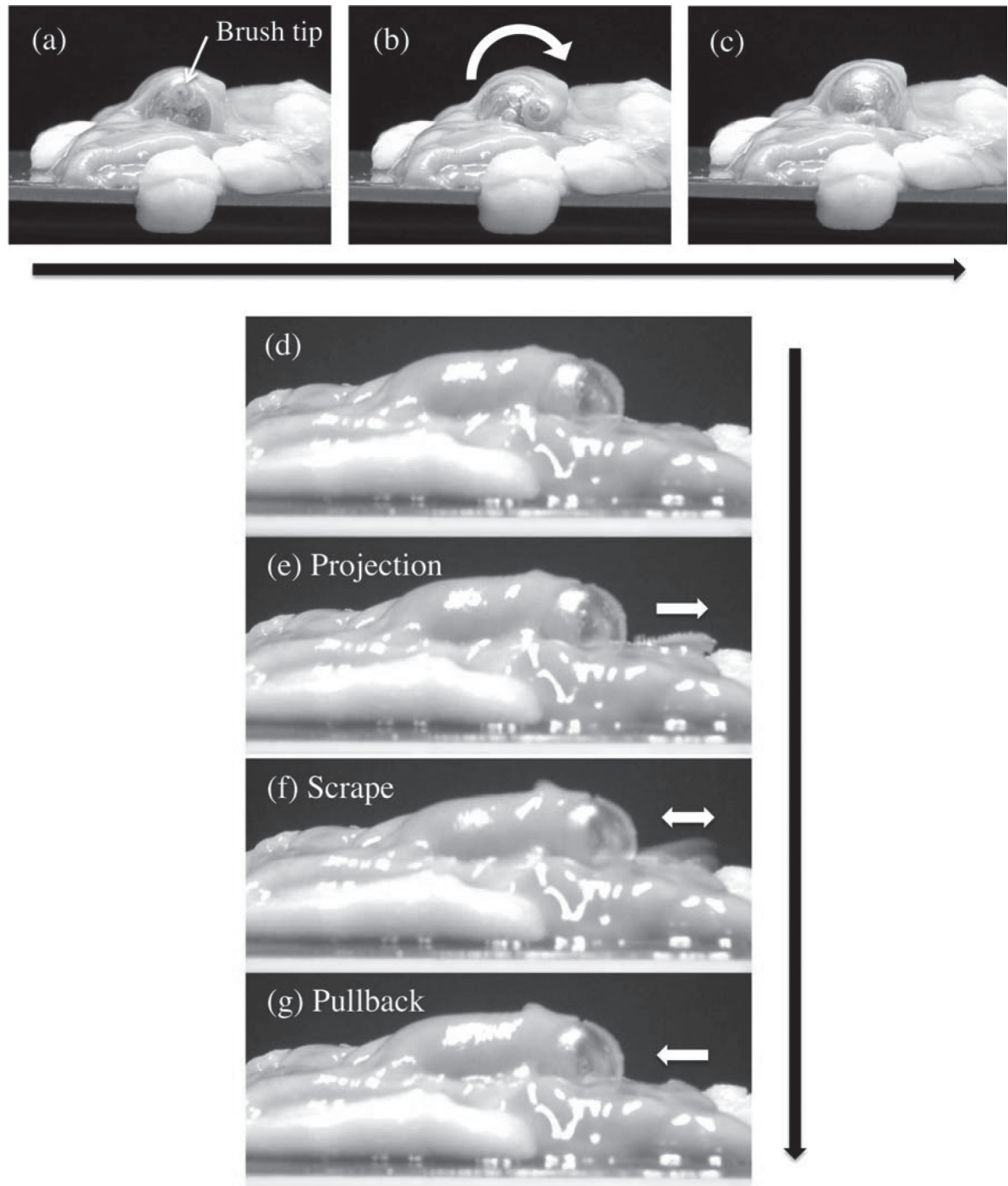

Fig. 12. Video capture images of the consecutive operation. The images (a)-(c) show the capsule rotation viewed from the $\mathrm{x}$-axial direction, and the imagnes $(\mathrm{d})-(\mathrm{g})$ show the operations of the brush.

up the Ni-Ti wire and locks the magnetic latch at the initial state, it is determined that the pullback is successful. As a result, the magnetic field strength required for the pullback of the brush was determined as more than $7 \mathrm{kA} / \mathrm{m}$.

\subsection{Consecutive operation test}

As described above, all actuation modes expected in the Chapter 2 have been individually achieved at relatively low magnetic fields. Finally, the consecutive operation test was conducted in the cow's small intestine. Table 1 shows the driving conditions for the consecutive operation. The magnetic field strength was determined by adding a margin to the experimental values. Figure 12 shows video capture images of the consecutive operation for the normal brush. The images (a)-(c) show the capsule rotation viewed 
Table 1

Driving conditions for consecutive operation

\begin{tabular}{lcl}
\hline Mode & Magnetic field strength $[\mathrm{kA} / \mathrm{m}]$ & Direction and frequency \\
\hline Capsule rotation & 7.0 (Normal brush) 9.5 (Improved brush) & Rotation $(\leqslant 1 \mathrm{~Hz})$ in yz-plane \\
$\begin{array}{l}\text { Projection } \\
\text { Scrape }\end{array}$ & 9.6 & Single DC pulse in positive x-direction \\
(Normal brush) & 6.0 & AC in z-direction $(\leqslant 30 \mathrm{~Hz})$ \\
$\begin{array}{l}\text { Scrape } \\
\text { (Improved brush) }\end{array}$ & $6.0 / 4.0-8.0$ & AC in z-direction $(\leqslant 30 \mathrm{~Hz}) / \mathrm{DC}$ in positive x-direction \\
Pullback & 7.2 & DC in negative x-direction \\
\hline
\end{tabular}

from the $\mathrm{x}$-axial direction, and the images $(\mathrm{d})-(\mathrm{g})$ show the operations of the brush viewed from the $\mathrm{y}$-axial direction. As is evident from these images, a sequence of the operations could be successfully performed.

\section{Conclusions}

A magnetically driven cytology brush has been proposed for the CE. It could provide four different actuations including projection/pullback/scrape modes for the brush and a rotation mode for the capsule body. Each actuation mode could be selected by the direction of the external magnetic fields. Furthermore, an additional small magnet attached to the brush tip enhanced the performance of the scrape mode. These results suggest the proposed magnetic actuator should be applicable for the $\mathrm{CE}$ as a diagnostic tool.

Our future work will include development of an anchoring mechanism to stay in a certain place against peristaltic movement of GI tract.

\section{Acknowledgement}

This work was supported by JSPS KAKENHI Grant Number 25420260.

\section{References}

[1] A. Menciassi, A. Moglia, S. Gorini, G. Pernorio, C. Stefanini and P. Dario, Shape memory alloy clamping devices of a capsule for monitoring tasks in the gastrointestinal tract, J. Micromech Microeng 15 (2005), 2045-2055.

[2] S. Park, K. Koo, Seoung M. Bang, J.Y. Park, S.Y. Song and D. Cho, A novel microactuator for microbiopsy in capsular endoscopes, J Micromech Microeng 18 (2008), 025032.

[3] F. Carpi and C. Pappone, Magnetic maneuvering of endoscopic capsules by means of a robotic navigation system, IEEE Trans Biomed Eng 56 (2009), 1482-1490.

[4] A. Chiba, M. Sendoh, K. Ishiyama, K.I. Arai, H. Kawano, A. Uchiyama and H. Takizawa, Magnetic actuator for a capsule endoscope navigation system, J Magn 12 (2007), 89-92.

[5] F. Munoz, G. Alici and W. Li, A review of drug delivery systems for capsule endoscopy, Adv Drug Deliv Rev 71 (2014), 77-85.

[6] M. Yamashita and T. Honda, Fabrication of magnetic microactuators for cytology brush built into capsule endoscope, Electr Commun JPN 98 (2015), 27-34. 